\title{
MENINGEOMA PÓS-RADIOTERAPIA
}

\author{
Erick de Souza Barboza', Alexandros Theodoros Panagopoulos', \\ Carmen Lúcia Penteado Lancellotti ${ }^{3}$, José Carlos Esteves Veiga ${ }^{4}$
}

\begin{abstract}
RESUMO - Os meningeomas são neoplasias derivadas das células aracnóideas, sendo sua origem ligada a alterações genéticas idiopáticas (deleção do braço longo do cromossomo 22), doenças predisponentes à sua formação e indução por radioterapia. Relata-se o caso de um homem de 50 anos com meningeoma pós-radioterapia diagnosticado 20 anos após o tratamento de um oligodendroglioma. Os critérios que suportam o diagnóstico de meningeoma induzido por radioterapia são discutidos, assim como é revisada a literatura pertinente ao assunto.
\end{abstract}

PALAVRAS-CHAVE: meningioma, radioterapia, oligodendroglioma.

\section{Radiation-induced meningioma}

ABSTRACT - Meningiomas are neoplasms derived from arachnoid cells with their origin linked to idiopathic genetic abnormalities (delection of the long arm of chromosome 22), predisposing diseases and radiotherapy induction. We report the case of a 50 years-old man radiation-induced meningioma 20 years after the diagnosis, surgical and radiation treatment of an oligodendroglioma. The supporting diagnostic criteria of radiation-induced meningiomas are discussed and the pertinent literature of the theme is revised.

KEY WORDS: meningioma, radiotherapy, oligodendroglioma.

Os meningeomas são neoplasias que podem surgir após irradiação do crânio, utilizada para lesões do escalpo ou intracranianas. Somente a radiação ionizante foi identificada como agente etiológico relacionado à ocorrência destas neoplasias ${ }^{1,2}$. O papel do trauma craniano, infecções virais e hormônios sexuais

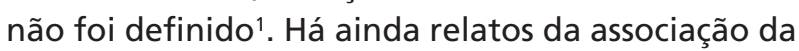
neurofibromatose tipo 2 e do câncer de mama com maior incidência de meningeomas ${ }^{1}$. O aparecimento de uma lesão (nova ou progressiva) alguns anos após a realização de radioterapia para neoplasia primária do sistema nervoso central (SNC) pode representar recorrência da neoplasia, radionecrose ou, raramente, neoplasia secundária ${ }^{3,4}$.

Relata-se um caso de meningeoma atípico pósradioterapia em paciente operado de oligodendroglioma, levando-se em consideração sua evolução pós-operatória no período de 20 anos; os seguintes critérios são avaliados: clínicos, radiológicos e histológicos da neoplasia secundária; tratamento e prognóstico.

\section{CASO}

Homem de 50 anos, branco, apresentou em dezembro de 1980 queixa de cefaléia e vômitos de início há 21 dias, com piora progressiva. Relatava crises convulsivas há 6 anos, estando desde então em uso de medicação anticonvulsivante, sem ter sido submetido a investigação clínica prévia. Ao exame neurológico apresentava apenas papiledema. A tomografia computadorizada craniencefálica (TC) mostrou lesão frontal esquerda compatível com glioma de baixo grau. Foi então submetido a craniotomia e lobectomia frontal esquerda (19/12/1980). O exame anátomo-patológico mostrou tratar-se de oligodendroglioma. Recebeu tratamento radioterápico convencional complementar com 5400 cGy (dose total em crânio), sendo encaminhado para seguimento ambulatorial. As TC subseqüentes não mostraram sinais de recorrência da neoplasia (Fig 1). O paciente não apresentava déficit neurológico, tendo recebido alta ambulatorial em 1993.

Em outubro de 2001, foi reinternado em nosso Serviço devido a quadro de distúrbio da linguagem com início há 06 meses e piora há dois meses associado a cefaléia holocraniana com aumento progressivo da intensidade há 20 dias. Ao exame neurológico, apresentava-se com disfasia de expressão e papiledema. A TC de crânio, nessa ocasião, evidenciou lesão isoatenuante fronto-parieto-temporal esquerda, com realce homogêneo e intenso à infusão de contraste. A ressonância magnética encefálica (RM) mostrava lesão extensa fronto-parieto-temporal esquerda, isointensa em T1, com realce homogêneo à infusão de contraste (ga-

\footnotetext{
Serviço de Neurocirurgia da Faculdade de Ciências Médicas da Santa Casa de São Paulo, São Paulo, SP, Brasil (SN-FCMSCSP): ${ }^{1}$ Médico Ex-residente do SN-FCMSCSP; ${ }^{2}$ Médico Assistente do SN-FCMSCSP; ${ }^{3}$ Doutora em Medicina, Prof ${ }^{a}$. Adjunta responsável pelo Serviço de Neuropatologia; ${ }^{4}$ Doutor em Medicina, Chefe do SN-FCMSCSP.
}

Recebido 3 Fevereiro 2006, recebido na forma final 19 Junho 2006. Aceito 17 Julho 2006.

Dr. Erich de Souza Barboza - Alameda Barros 66 / 213 - 01232-000 São Paulo SP - Brasil. 


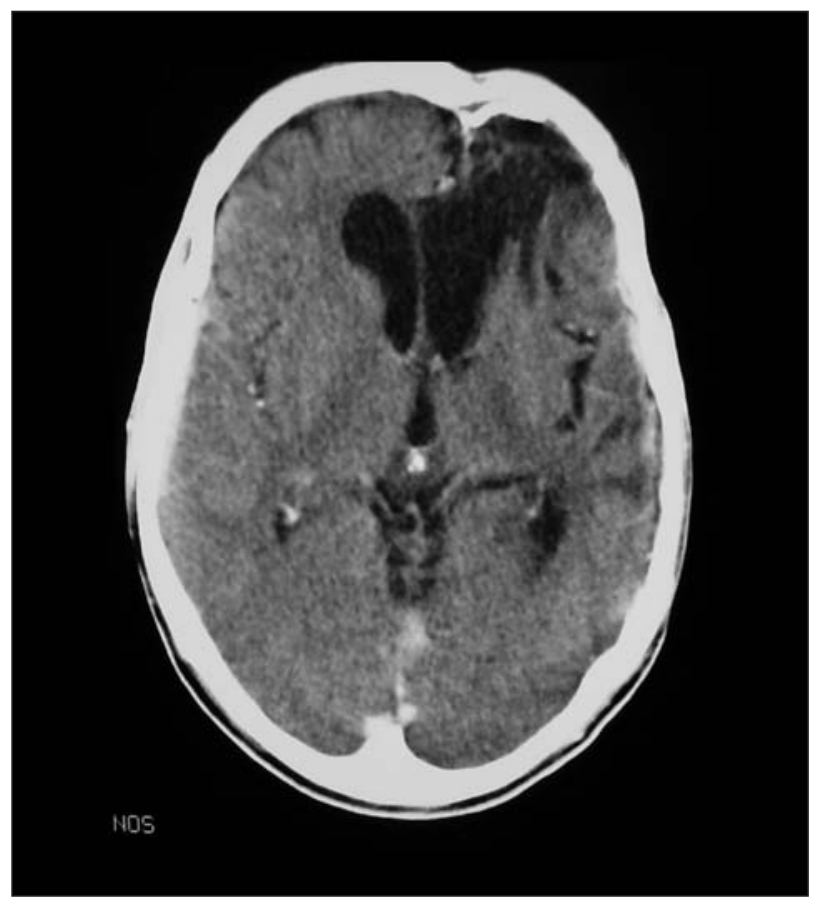

Fig 1. TC com contraste realizada em 26/12/ 1991. Não se observam sinais de neoplasia; há área de gliose frontal esquerda compatível com status pós-operatório, 11 anos após a intervenção cirúrgica.

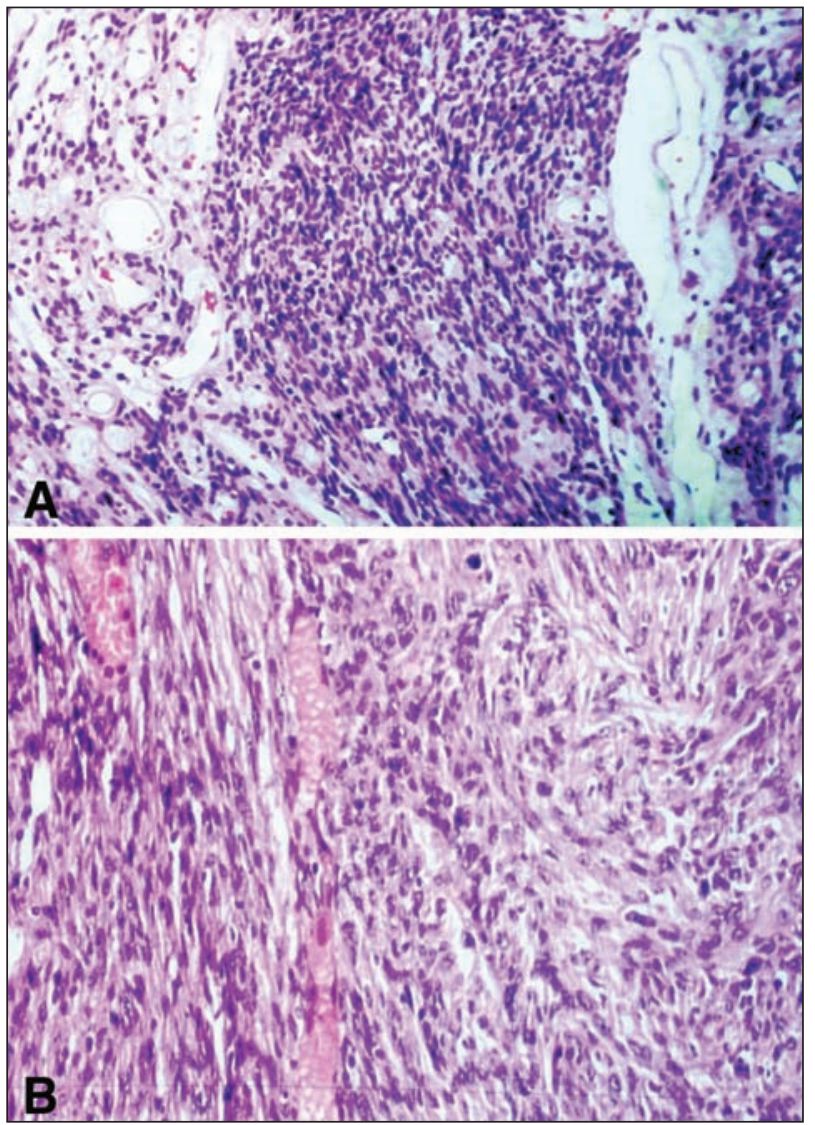

Fig 3. (A) Área hipercelular à direita. HE X100; (B) Hipercelularidade, polimorfismo celular e nuclear, mitoses. HE X200.

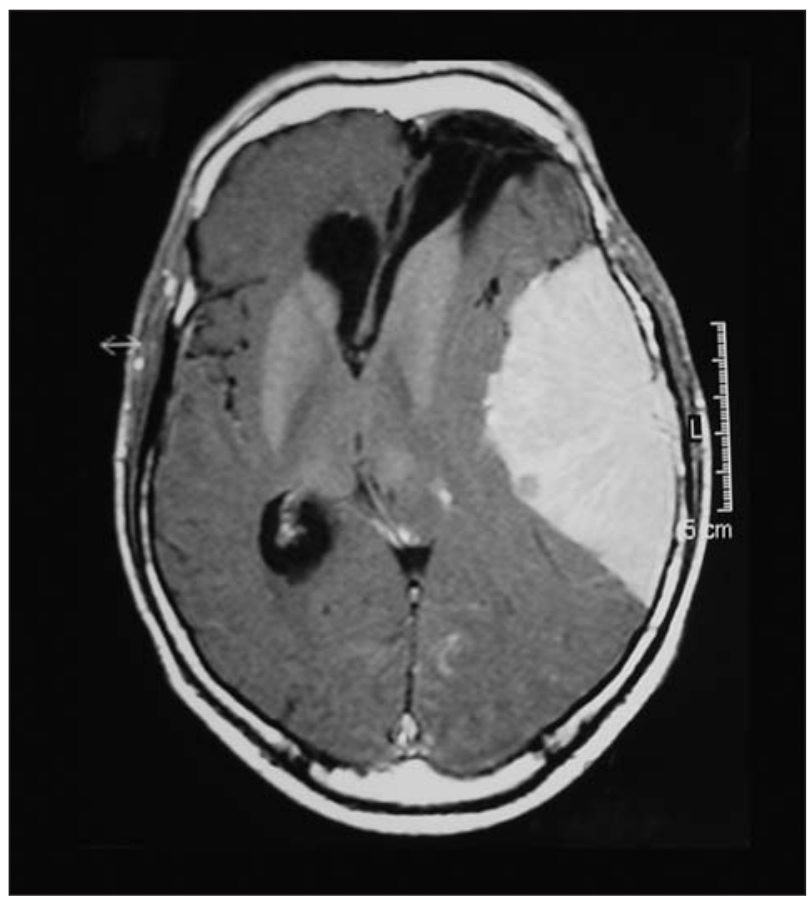

Fig 2. RM (T1 com contraste) mostrando lesão expansiva frontoparieto-temporal, com realce homogêneo à infusão de contraste (gadolíneo).

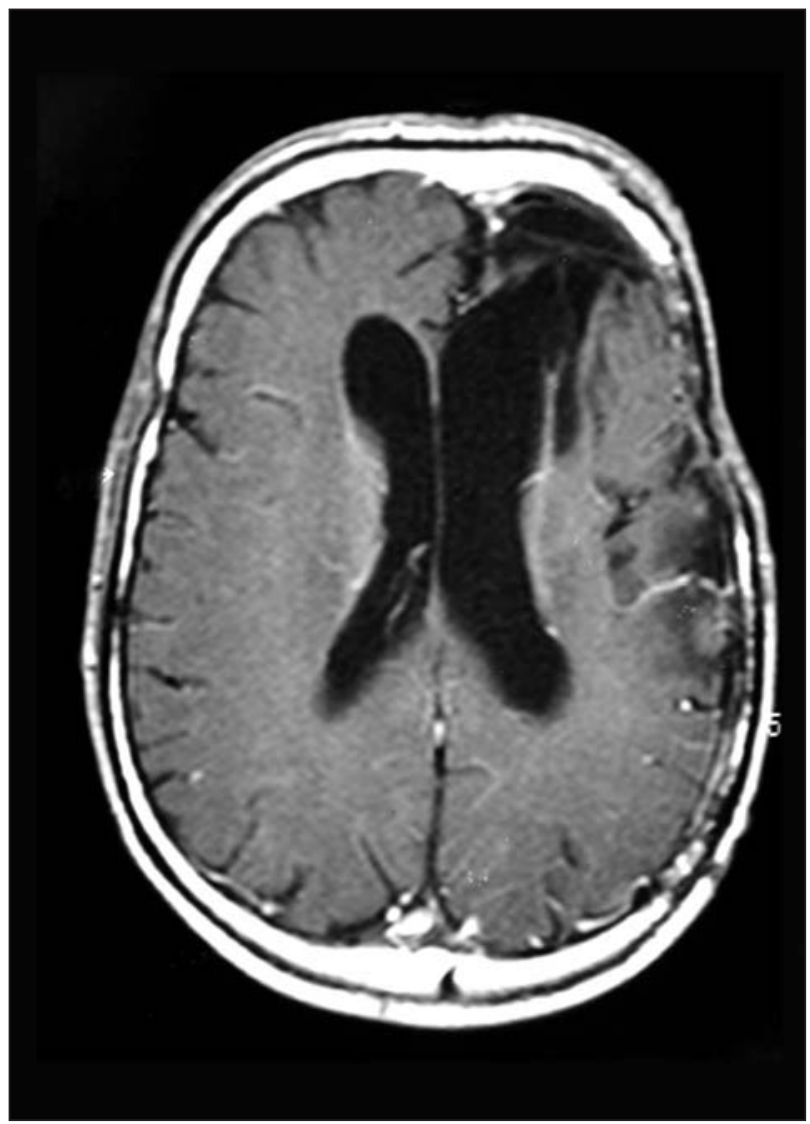

Fig 4. RM (T1 com contraste) realizada em 11/12/2002, com ausência de sinais de neoplasia. 
dolíneo) e hiperintensa em T2 (Fig 2). Foi submetido a craniotomia e exérese microcirúrgica da neoplasia em 05/11/ 2001, com ressecção completa (Simpson I) 5 . O resultado do exame anátomo-patológico foi de meningeoma atípico (Figs 3A-B).

No pós-operatório, manteve o distúrbio da linguagem, estando atualmente em acompanhamento ambulatorial. Os exames de neuroimagem pós-operatórios (TC e RM) confirmaram a exérese completa da neoplasia, sem evidências de recorrência ou de surgimento de outras lesões (Fig 4).

\section{DISCUSSÃO}

Neoplasias intracranianas podem se desenvolver como conseqüência de radioterapia, especialmente os meningeomas e os tumores gliais. Nestas condições, são considerados tumores secundários. De acordo com Russel e Rubinstein, citados por Dweik ${ }^{6}$, as células gliais e meníngeas são as mais vulneráveis à transformação neoplásica no SNC. São situações raras; porém, com o aumento da sobrevida de pacientes com doenças neoplásicas encefálicas e o amplo uso da radioterapia, tendem ao aumento de sua freqüência ${ }^{7,8}$.

O primeiro caso de meningeoma induzido por radioterapia em altas doses foi descrito por Mann em $1953^{7,8}$. Desde então, tumores intracranianos induzidos por radioterapia têm sido descritos na literatura e incluem meningeomas, sarcomas e gliomas ${ }^{7,9-12}$. Meningeoma é mais freqüente após baixas doses, enquanto os gliomas e sarcomas são mais comuns após altas doses ${ }^{12}$. Estima-se que, dentre os tumores induzidos por radioterapia, os meningeomas são cinco vezes mais freqüentes que os sarcomas e glio$\operatorname{mas}^{8,13}$.

Comumente, os meningeomas aparecem na quinta e sexta décadas de vida, mas os meningeomas induzidos por radioterapia ocorrem em pacientes mais jovens ${ }^{6,13}$. O pico de incidência destes meningeomas é na terceira década de vida ${ }^{8}$.

As meninges das crianças parecem ser particularmente sensíveis aos efeitos da radiação, uma vez que grande parte desses meningeomas foi observada em pacientes submetidos a radioterapia na infância ${ }^{6,14}$. A radiação ionizante provavelmente altera as bases do DNA, especificamente o braço longo do cromossomo 22 , levando a mutações que afetam os oncogenes e genes supressores tumorais ${ }^{2,7,10}$. Estudos genéticos mostram diferentes alterações em meningeomas esporádicos, como as mutações no NF2 e a deleção no cromossomo 22q. Estudos comparativos das anormalidades cromossômicas em meningeomas esporádicos e radioinduzidos revelam pequenas diferenças, que provavelmente não tenham papel importante na gênese deste subtipo de tumor ${ }^{14-16}$. Foi demonstrado que glioblastomas e sarcomas induzidos por radioterapia possuem mutações do gene $p 53^{10}$. A radiação poderia também causar dano ao estroma e inflamação meníngea, determinando mutações nos processos regenerativos ${ }^{7}$.

A histopatologia dos meningeomas induzidos por radioterapia foi definida da seguinte maneira por Rubinstein: alta celularidade, núcleos pleomórficos e presença de vacúolos nucleares claros $^{6}$. Os meningeomas atípicos são relativamente freqüentes entre os meningeomas induzidos por radioterapia ${ }^{8,10,13}$. Relatam-se taxas de $76 \%$ classificados como benignos e $24 \%$ classificados como atípicos/anaplásicos ${ }^{13}$. Estes últimos são associados a maior taxa de recorrência, o que mostra tendência a um comportamento biológico mais agressivo ${ }^{13}$. Histologicamente, podem apresentar crescimento densamente celular laminar, nucléolos proeminentes, figuras de mitose, pleomorfismo excessivo e necrose, com tendência maior à recorrência ${ }^{8}$. Em meningeomas induzidos por radioterapia tem-se uma maior imunorreatividade para receptores de estrógenos e progesterona. Para serem considerados secundários, os meningeomas pós-radioterapia obedecem aos seguintes critérios: 1) devem localizar-se no leito irradiado; 2) não deve haver lesão prévia à radioterapia; 3) histologicamente, o tumor deve ser diferente do tumor primário; 4) intervalo razoável entre o surgimento do segundo tumor e a realização da radioterapia (geralmente acima de cinco anos); 5) ausência de condições que favoreçam o surgimento de meningeomas (facomatoses, xeroderma pigmentoso, imunodeficiência, retinoblastoma, síndrome do nevo de células basais) ${ }^{8}$.

Kandel e colaboradores classificaram os meningeomas induzidos por radioterapia em dois grupos: 1) pós-radioterapia em baixas doses (<10 Gy) e 2) pósradioterapia em altas doses $(>20 \mathrm{~Gy})^{6,10}$. Os primeiros geralmente ocorreram em pacientes submetidos a tratamento de tinea capitis do couro cabeludo e os últimos em pacientes tratados devido a neoplasias cerebrais primárias ${ }^{6,10}$. Para outros autores, há ainda um terceiro grupo, formado por pacientes que receberam doses intermediárias de radiação ${ }^{6,18}$.

O intervalo de tempo entre o surgimento do meningeoma secundário e o tratamento radioterápico depende da dose de radiação recebida. Em pacientes que receberam baixas doses (<10 Gy) é, em média 35,2 anos e 18,7 anos naqueles que receberam altas doses (>20 Gy) 7 . Mack e Wilson observaram um período de latência de 27 anos para doses moderadas 
$(10-20 \mathrm{~Gy})^{19}$. Estes diferentes períodos de latência sugerem que o maior dano ao cromossomo causado por doses maiores de radiação induz à perda mais rápida do mecanismo de controle celular e à expressão fenotípica neoplásica mais precoce ${ }^{7}$. As características clínicas e anátomo-patológicas dos meningeomas pós-radioterapia em altas doses são: 1) crianças são mais susceptíveis; 2 ) predominância no sexo feminino, diferentemente da predominância masculina nos meningeomas induzidos por radioterapia em baixas doses; 3) pico de incidência na terceira década de vida; 4) freqüentemente são tumores atípicos e que recidivam ${ }^{8,13}$.

Para neoplasias cerebrais primárias, assim como induzidas por radioterapia, o tratamento mais eficiente ainda hoje, considerado padrão ouro, é a ressecção cirúrgica combinada à radioterapia ${ }^{10}$. No entanto, nos tumores induzidos por radioterapia, o tratamento convencional encontra-se comprometido devido às doses moderadamente altas recebidas previamente ${ }^{10}$. Nesses casos, existe a opção de se tratar através de irradiação localizada em baixas doses por meio da radiocirurgia ${ }^{20-23}$.

O tratamento de escolha nos casos de meningeomas induzidos por radioterapia é a ressecção completa da lesão, com taxa de recorrência nas grandes séries que varia de $38-50 \%$ em cinco anos ${ }^{24,25}$. Devido à maior propensão desses tumores à recorrência, recomenda-se uma margem ampla de ressecção óssea e da dura-máter durante o procedimento cirúrgico (grau I da escala de Simpson)3,5, podendo ser realizado tratamento complementar com radiocirurgia ${ }^{20-25}$. Recentemente, foram relatados bons resultados a longo prazo com o uso de hidroxiuréia em meningeomas benignos irressecáveis e recorrentes, porém sua eficácia não é significativa para meningeomas malignos e atípicos ${ }^{4,25}$. Os meningeomas benignos irressecáveis, os recorrentes, assim como os malignos e atípicos também podem ser tratados com o uso de interferon $\alpha-2 B^{1}$. Desta forma, obteve-se estabilização do tamanho do tumor ou até mesmo sua regressão". O risco de formação de neoplasias radioinduzidas após radiocirurgia é desconhecido, porém em pacientes que receberam radioterapia e radiocirurugia este risco pode aumentar ${ }^{26}$.

\section{REFERÊNCIAS}

1. Kaba SE, Demonte F, Bruner JM, et al. The treatment of recurrent unresectable and malignant meningiomas with interferon alpha- $2 \mathrm{~b}$ Neurosurgery 1997;40:271-275.
2. Pagni CA, Canavero S, Fiocchi F, Ponzio G. Chromosome 22 monosomy in a radiation-induced meningioma. Ital J Neurol Sci 1993;14:377-379.

3. Harrison MJ, Wolfe DE, Lau TS, Mitnick RJ, Sachdev VP. Radiationinduced meningiomas: experience at the Mount Sinai Hospital and review of the literature. J Neurosurg 1991;75:564-574.

4. Mason WP, Gentili F, Macdonald DR, Hariharan S, Cruz CR, Abrey LE. Stabilization of disease progression by hydroxyurea in patients with recurrent or unresectable meningioma. J Neurosurg 2002;97:341-346.

5. Simpson D. The recurrence of intracranial meningiomas after surgical treatment. J Neurol Neurosurg Psychiatry 1957;20:22-39.

6. Dweik A, Maheut-Lourmiere J, Lioret E, Jan M. Radiation-induced meningioma. Child's Nerv Syst 1995;11:661-663.

7. Salvati M, Cervoni L, Puzzilli F, Bristot R, Delfini R, Gagliardi FM. High-dose radiation-induced meningiomas. Surg Neurol 1997;47: 435-442.

8. Salvati M, Cervoni L, Caruso R, Delfini R, Gagliardi FM. High-dose radiation-induced meningiomas in elderly. Neurosurgery 1996;19: 81-83.

9. Deutsch M, Rosenstein M, Figura JH. Meningioma after radiotherapy for Hodgkin's disease. Am J Clin Oncol 1999;22:361-363.

10. Pollak L, Walach N, Gur R, Schiffer J. Meningiomas after radiotherapy for tinea capitis: still no history. Tumori 1998;84:65-68.

11. Sadetzki S, Flint-Richter P, Ben-Tal T, Nass D. Radiation-induced meningioma: a descriptive study of 253 cases. J Neurosurg 2002;97: 1078-1082.

12. Starshak RJ. Radiation-induced meningioma in children: report of two cases and review of the literature. Pediatr Radiol 1996;26:537-541.

13. Strojan P, Popovic M, Jereb B. Secondary intracranial meningiomas after high-dose cranial irradiation: report of five cases and review of the literature. Int J Radiation Oncol Biol Phys 2000;48:65-73.

14. Whittle IR, Smith C, Navoo P, Collie D. Meningiomas. Lancet 2004;363 (9420):1535-1543.

15. Gosztonyi G, Slowik F, Pasztor E. Intracranial meningiomas developing at long intervals following low-dose $\mathrm{X}$-ray irradiation of the head. J Neurooncol 2004;70:59-65.

16. De Tommasi A, Occhiogrosso M, De Tommasi C, Cimmino A, Sanguedolce F, Vailati G. Radiation-induced intracranial meningiomas: review of six operated cases. Neurosurg Rev 2005;28:104-114.

17. Alexander MJ, Desalles AAF, Tomiyasu U. Multiple radiation-induced intracranial lesions after treatment for pituitary adenoma. J Neurosurg 1998;88:111-115.

18. Mack EE, Wilson CB. Meningiomas induced by high-dose cranial irradiation. J Neurosurg 1993;79:28-31.

19. Debus J, Wuendrich M, Pirzkall A, et al. High efficacy of fractionated stereotactic radiotherapy of large base-of-skull meningiomas: longterm results. J Clin Oncol 2001;19:3547-3553.

20. Kobayashi T, Kida Y, Mori Y. Long-term results of stereotactic gamma radiosurgery of meningiomas. Surg Neurol 2001;55:325-331.

21. Nishio S, Morioka T, Inamura T, et al. Radiation-induced brain tumours: potential late complications of radiation therapy for brain tumours. Acta Neurochir 1998;140:763-770.

22. Shafron DF, Friedman WA, Buatti JM, Bova FJ, Mendenhall WM. Linac radiosurgery for benign meningiomas. Int J Radiat Oncol Biol Phys 1999;43:321-327.

23. Jääskeläinen J, Haltia M, Servo A. Atypical and anaplastic meningiomas: radiology, surgery, radiotherapy, and outcome. Surg Neurol 1986;25: 233-237.

24. Mahmood A, Caccamo DV, Tomecek FJ, et al. Atypical and malignant meningiomas: a clinicopathological review. Neurosurgery 1993;33: 955-959.

25. Schrell UMH, Rittig MG, Anders M, et al. Hydroxyurea for treatment of unresectable and recurrent meningiomas: II. Decrease in the size of meningiomas in patients treated with hydroxyurea. J Neurosurg 1997; 86:840-844.

26. Mciver JI, Pollock BE. Radiation-induced tumor after stereotactic radiosurgery and whole brain radiotherapy: case report and literature review. J Neurooncol 2004;66:301-305. 